\title{
Timing Nutriceuticals?
}

\author{
Franz Halberg ${ }^{1, *}$, Germaine Cornélissen ${ }^{1}$ and R.B. Singh ${ }^{2}$ \\ ${ }^{1}$ Halberg Chronobiology Center, University of Minnesota, Minneapolis, MN 55455, USA \\ ${ }^{2}$ Halberg Hospital and Research Institute, Centre of Nutrition and Heart Research, Civil Lines, Moradabad-10, UP, \\ India
}

\begin{abstract}
Evidence on time-dependent effects of drugs and nutrition is succinctly reviewed in order to illustrate and advocate investigations of the timing of nutriceuticals. Emphasis is placed on the merits of coordinated, individually, inferentially, statistically examined sets of N-of-1 studies.
\end{abstract}

Keywords: Chronotherapy, coenzyme Q10, marker rhythms, Timing.

\section{BACKGROUND}

An article just published by Deanna M. Arble et al. [1,2] provides added evidence that feeding at the "wrong time" can lead to weight gain. Data related to these recent findings on mice had already been shown in humans in 1973 [3], as illustrated in Fig. (1) (top) with follow-ups (Fig. 2-4) [4-6]. As seen in Fig. (1) (bottom), in the mouse, the timing of food intake can contribute to the difference between death and survival [7]. Such a difference had also been documented and tabulated for other agents, including drugs for high blood pressure (BP) $[8,9]$ and the irradiation of perioral tumors [10, 11] (Fig. 5-6), among many other treatments [12-15]. A start with nutriceuticals indicates that in a person investigated by varying the administration times of Coenzyme Q10 (CoQ10), the circadian stage played an important role (Fig. 7-8) [16, 17].

In the experimental laboratory, CoQ10 treatment was found to be associated with a statistically significant reduction in toxicity from doxorubicin [18]. In humans, several of us determined, each with an N-of-1 approach, whether CoQ10 softgels (Q-Gel) supplementation, kindly provided by the Tishcon Corporation (Westbury, NY), affect BP, and if so, what the optimal stage of Q-Gel administration may be. Clinical trials of CoQ10 in the management of high BP reportedly concluded that CoQ10 is associated with a substantial reduction in both systolic (S) and diastolic (D) BP, without the side effects commonly seen with conventional therapy, a proposal by one of us (RBS) [19, 20], awaiting longitudinal validation. Putative beneficial effects of CoQ10 may not invariably be found on an individual basis during spans of days or even of single weeks rather than months because BP is highly variable and can be influenced by a host of factors other than those being investigated.

\section{N-OF-1 RESULTS IN COQ10}

A clinically healthy woman (GC, 55 years of age) monitored her BP around the clock at 30-minute intervals with only few

\footnotetext{
*Address correspondence to this author at the Halberg Chronobiology Center, University of Minnesota, Minneapolis, MN 55455, USA;

Tel/Fax: 0091591 2417437; E-mail: halbe001@tc.umn.edu
}

interruptions for several months prior to the start of Q-Gel administration, using the TM-2430 monitor from A \& D (Tokyo, Japan). Starting March 13, 2005, GC took daily doses of Q-Gel (100 mg) during 6 weeks. During week 1, QGel was taken upon awakening; during weeks 2-5, it was taken $3.5,7,10.5$ and 14 hours after awakening, and during week 6 it was taken 17.5 hours after awakening or bedtime. The last 6 weeks of around-the-clock half-hourly monitoring of BP prior to the start of treatment were used as reference. Data during each of these 12 weeks were analyzed by cosinor to obtain estimates of the MESOR and of the circadian double amplitude (measure of the predictable extent of daily change) and acrophase (measure of the timing of overall high values recurring in each cycle). Student t-tests were used to compare the MESOR and circadian amplitudes of SBP and DBP between the 6 weeks of Q-Gel supplementation and the preceding 6 weeks without treatment. The SBP and DBP MESORs and circadian amplitudes during Q-Gel supplementation were also assigned to the circadian stage of treatment administration to assess any circadian-stage dependent effect of treatment, using the single cosinor method (Fig. 7-8).

A circadian rhythm in SBP and DBP was invariably demonstrated during each of the 12 weeks of study $(\mathrm{P}<0.001)$. As compared to the reference span, $\mathrm{Q}-\mathrm{Gel}$ was associated with a reduction of the circadian double amplitude of both SBP (from 40.5 to $30.9 \mathrm{mmHg}$; Student t $=5.005$; P $<0.001$ ) and DBP (from 24.8 to $18.5 \mathrm{mmHg}$, Student $\mathrm{t}=$ $4.725, \mathrm{P}<0.001)[16,17]$. This effect was circadian stagedependent (SBP: $\mathrm{P}=0.043$; DBP: $\mathrm{P}=0.012$ ), the largest reduction in circadian amplitude being associated with CoQ10 supplementation in the evening (around 14 hours after awakening) [16, 17].

Whether or not the observation in GC can be extended to more subjects, the N-of- 1 approach based on inferential statistics does apply to tests, e.g., of CoQ10 supplementation that may serve as a nutriceutical intervention to treat a circadian BP overswing (or CHAT, short for Circadian Hyper-Amplitude-Tension, a risk of stroke greater than a high BP). Notably in the absence of MESOR-hypertension, an active nutriceutical may be preferred as anti-hypertensive 


\section{Timing of calories determines outcomes (gain vs. loss in weight, top; death vs. survival, bottom)}
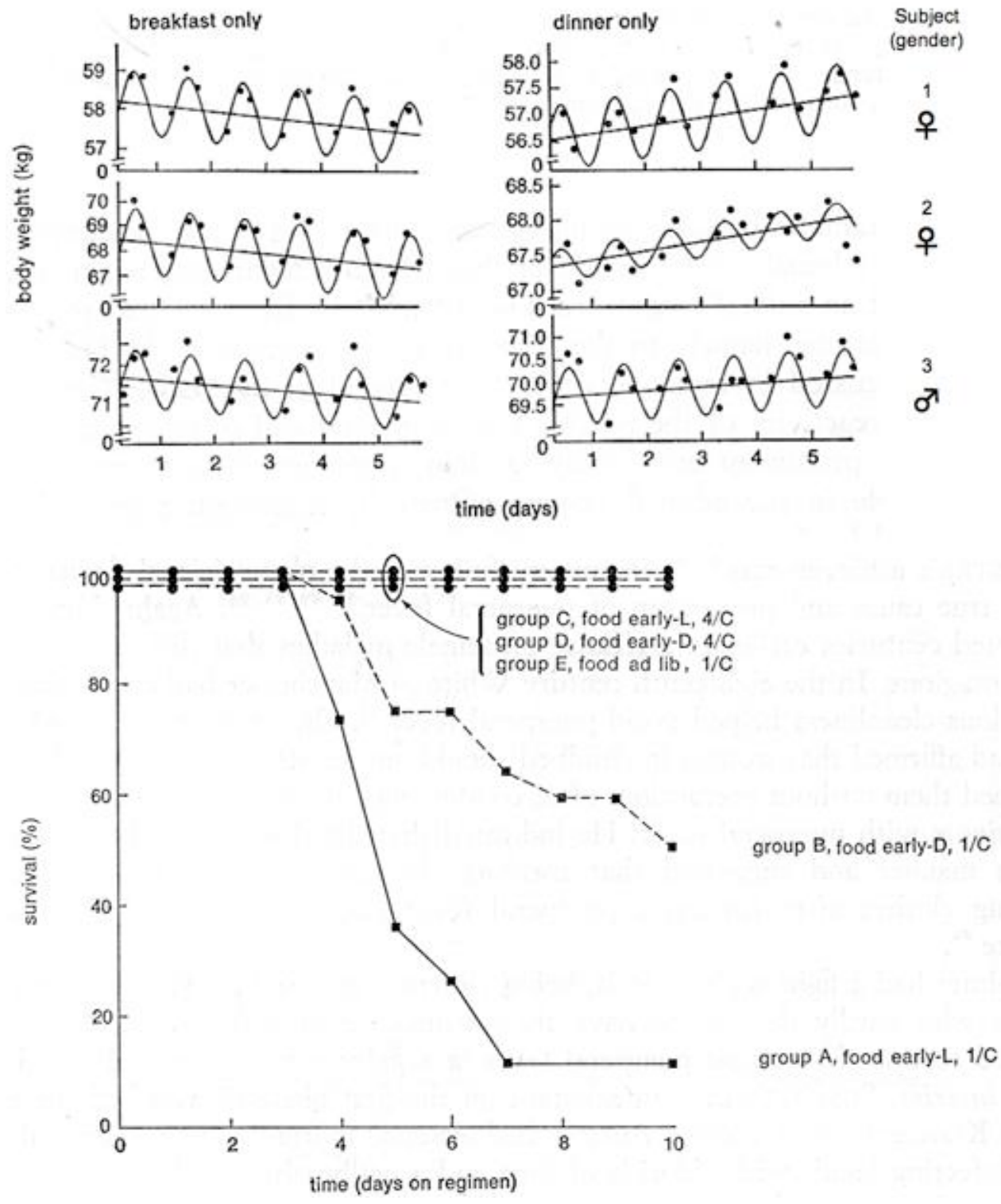

Fig. (1). Top: Three subjects consumed a single meal (2000 cal) per day, either in the morning (left) or in the evening (right). A cosine function with a period of 24 hours and a linear trend fitted concomitantly to the data were statistically significant 5 times (out of 6 ); P<0.05 for non-zero slope of trend 4 times out of 5 [6]. Results from more rigorous and extensive study on additional subjects over longer spans with control of mental and physical activities support, ceteris paribus, the finding of a relative body weight loss on breakfast.

Bottom: Survival of mice dependent on housing conditions and timing of single daily meal. Young male BALB/c mice kept on LD12:12 lighting regimen with 4-hour span of daily food accessibility. Initial group sizes: $A=19, B=20, C=16, D=16, E=20 ; 1 / C$ : housed 1 per cage; 4/C: housed 4 per cage. In this case, density per cage (allowing cuddling) represents an advantage rather than a disadvantage, a point of chronoecology. Experiment set up in Minnesota after reading of many deaths associated with lack of food, lack of clothing and cool weather among the palm trees of Benares on the Ganges. (C) Halberg.

medication. Clinical trials should be set up to see whether the results obtained on GC are more generally applicable. A chronobiologic design, such as the one used herein, is advocated so that any individual differences in response can be assessed. For so doing, the chronomically-assessed longitudinal monitoring of BP is critical. Automatic monitors can be obtained with a large reduction in price, with analyses, by participating in a project on The BIOsphere and the Cosmos
(BIOCOS) (by writing to corne001@umn.edu) in exchange for the data. Analyses are usually available free of charge from corne001@umn.edu and the Phoenix Project (www. phoenix.tc-ieee.org), a group of volunteering members of the Institute of Electrical and Electronics Engineers, which plans to place the software free of charge on the Internet and is working on an inexpensive, cuffless BP monitor, an urgent goal and a challenge for industry. 
A

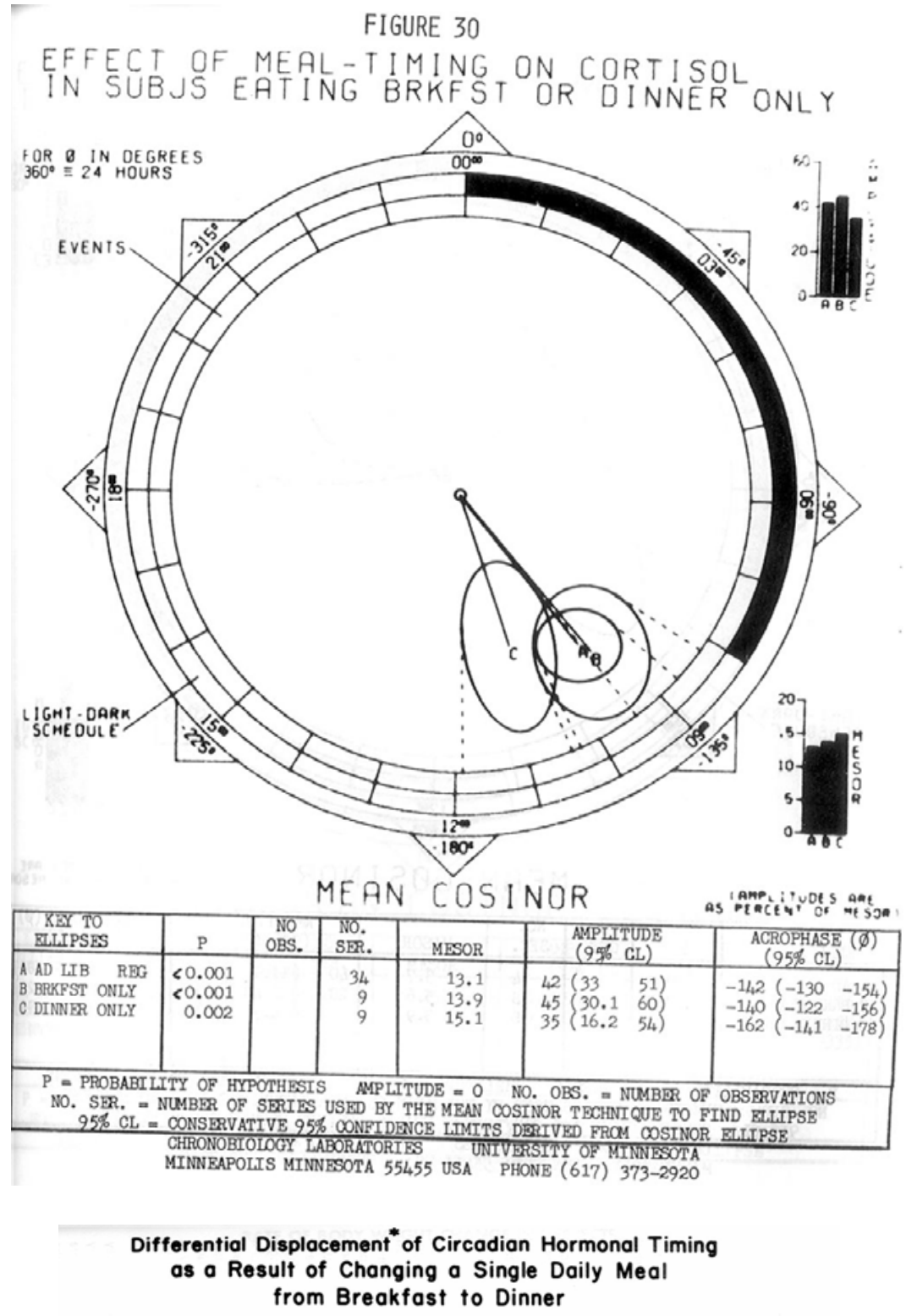

$\mathbf{B}$

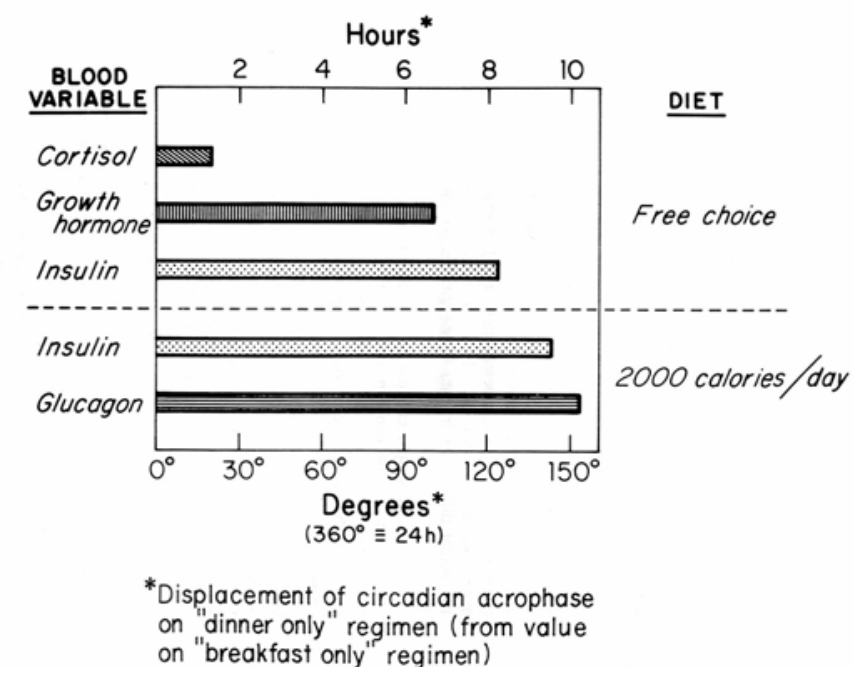

Fig. (2). (A). Effect of single meal as breakfast- or dinner-only on the circadian cortisol rhythm. (C) Halberg. (B). Different displacement of circadian timing for different (hormonal and other) variables as a result of changing a single daily meal from breakfast to dinner. Whereas the circadian rhythm of circulating cortisol is only slightly affected by the timing of a single daily meal, considerable phase-shifts are observed for the case of growth hormone, insulin, and glucagon. () Halberg. 
A

MEAL-TIMING AND BODY WEIGHT (BW)

Individual Weight Change Depending on Whether Single Daily Meal Either Free-Choice or Fixed - Eaten as Breakfast (B) or Dinner (D)*

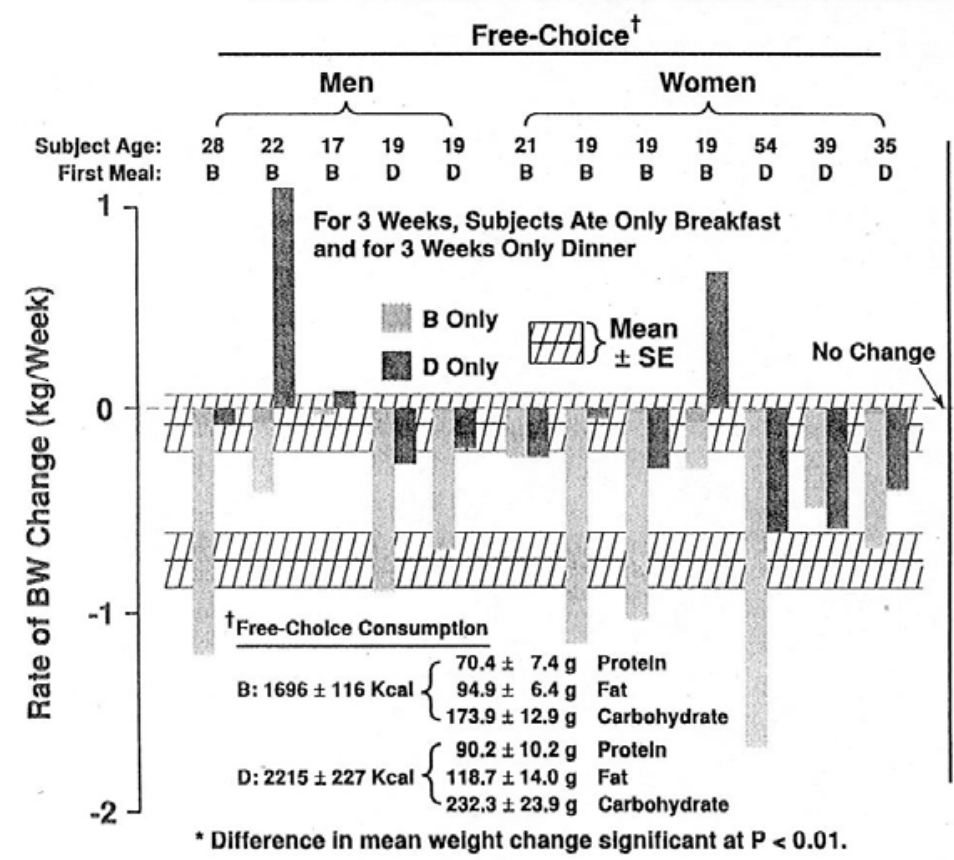

Fixed $2000 \mathrm{Kcal}$ Meal

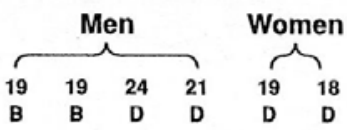

Single Fixed Meal/Day Eaten as Breakfast for One Week and as Dinner for Another

Difference in mean weight change significant at $P<0.01$.
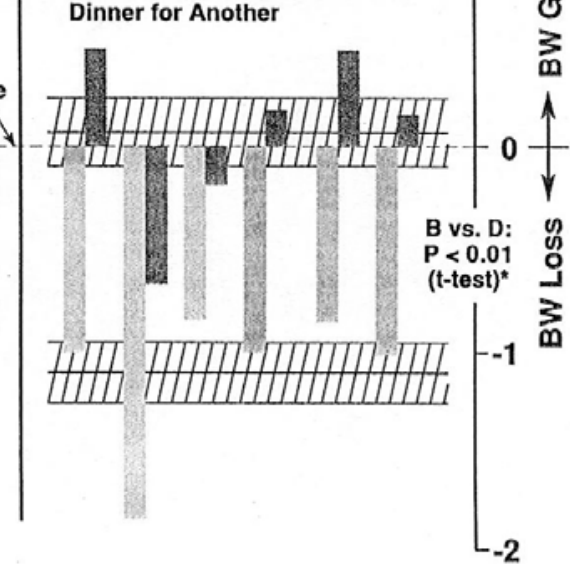

CC $10 / 93$

B

MEAL-TIMING AND BODY WEIGHT

Relative Body Weight Loss for Individuals Consuming

Single Daily Meal (Free-Choice or Fixed) as Breakfast (B) or Dinner (D)*

Free-Choice
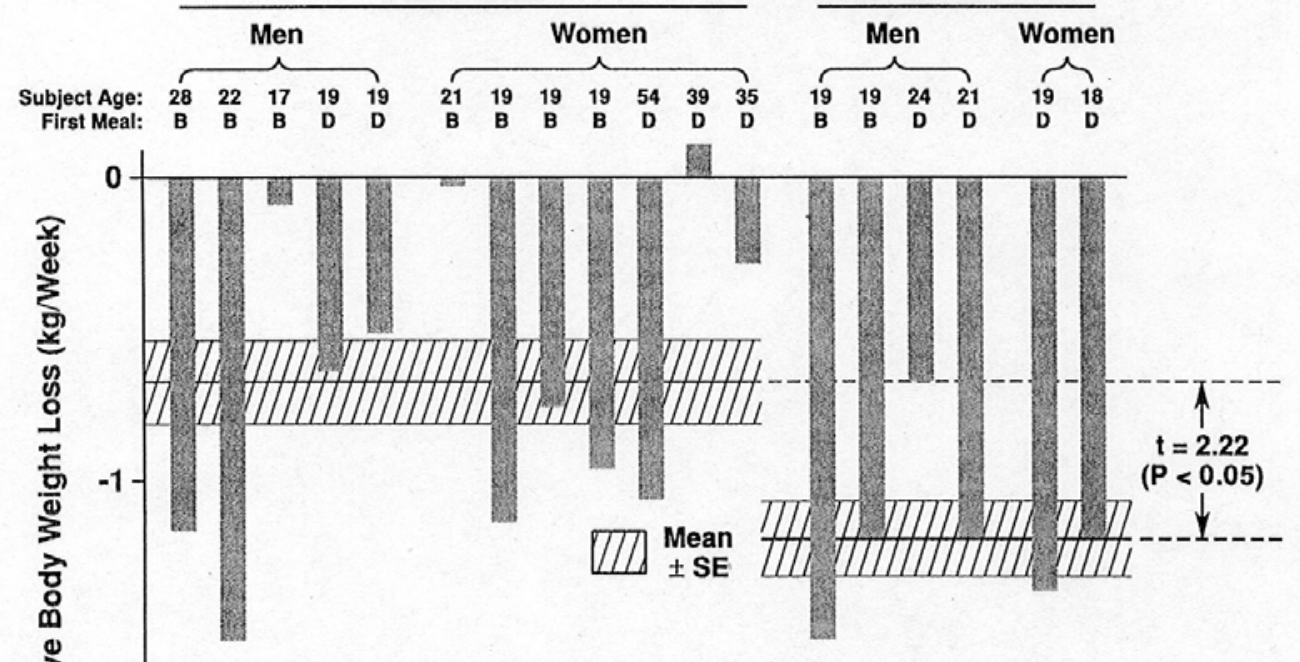

*Welght change (kg/week) on D subtracted from that on B; In study on single free-cholce meal/day, subjects ate only breakfast for 3 weeks and only dinner for 3 weeks; In study on flxed $2000 \mathrm{Kcal}$ single meal/day, subjects ate only breakfast for one week and only dinner for one week. Note that mean relative weight loss is greater on fixed than on free-choice meal.

Fig. (3). (A). Consuming a single daily meal as breakfast-only (light bars) vs. dinner-only (dark bars) affects the direction and/or rate of body weight change. The single daily meal was 2,000 kcal given for 7-day spans. Breakfast (B); dinner (D). (C) Halberg. (B). Individual (vertical columns) and average (horizontal slashed bars) summarizing results in Fig. (3A). With one exception, there was a relative body weight loss on breakfast vs. dinner. Only one volunteer gained weight on breakfast vs. dinner. Overall, the difference in relative body weight loss on breakfast (B) vs. dinner (D) is statistically significant $(\mathrm{P}<0.05)$, whether a fixed 2,000 kcal meal or a single free-choice meal is consumed. Weight change $(\mathrm{kg} / \mathrm{wk})$ on D subtracted from that on B. In the study on one free-choice meal per day, subjects ate only breakfast for 3 weeks and only dinner for 3 weeks. In the study on one fixed 2,000 kcal meal per day, subjects ate only breakfast for one week and only dinner for one week. Note that mean relative weight loss is greater on fixed than on free-choice meal. (c) Halberg. 


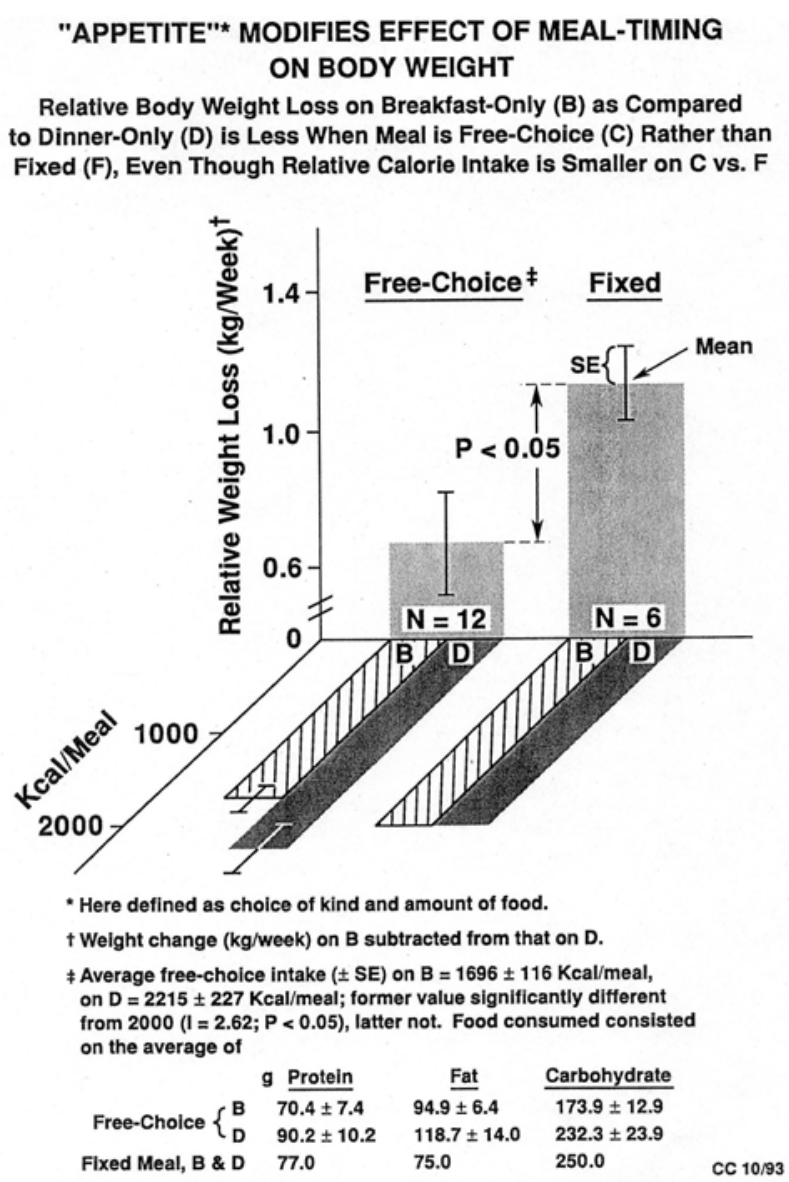

Fig. (4). Appetite (here defined as choice and amount of food) modifies the effect of meal timing on body weight. Relative body weight loss on breakfast-only (B) as compared to dinner-only (D) is less when meal is free-choice rather than fixed. Therefore, to minimize body weight loss when a single meal is consumed, it may be preferable not to fix the ration but to offer a choice. An overall summary of relative body weight loss on breakfast-only vs. dinner-only in the two studies described in Figs. (3A) and (3B) indicates that the decrease in relative body weight was more pronounced when a fixed 2,000-kcal meal was imposed than when volunteers could choose what they ate. (C) Halberg.

Better than Doubling Survival Achieved by Chronotherapy (CRx) *

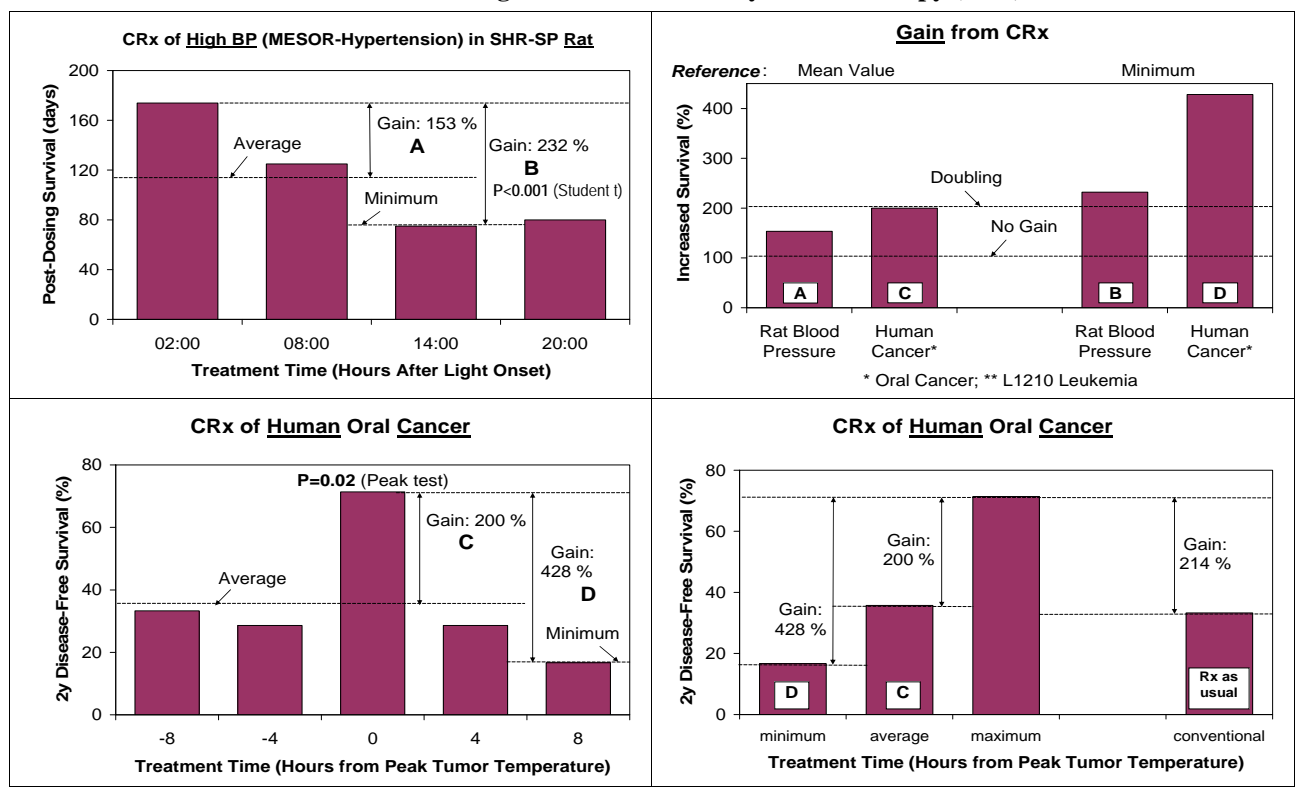

* In the case of blood pressure with $1 \mathrm{mg} / \mathrm{kg} /$ day Temocapril in salt-loaded stroke-prone Okamoto (SHR-SP) rats (top left) and by radiation of human cancers of the oral cavity timed by marker rhythmometry (of tumor temperature) (bottom left); Therapeutic gain assessed by comparing response at best time with average response or response at worst time (top right), or in the case of chronoradiotherapy, also with response to conventional treatment administered with timing during "regular hours" by convenience (bottom right).

Fig. (5). Chronotherapy results on rats (top) $[8,9]$ and humans (bottom) $[9,10]$. ( ) Halberg. 


\section{Gain from Chronotherapy}

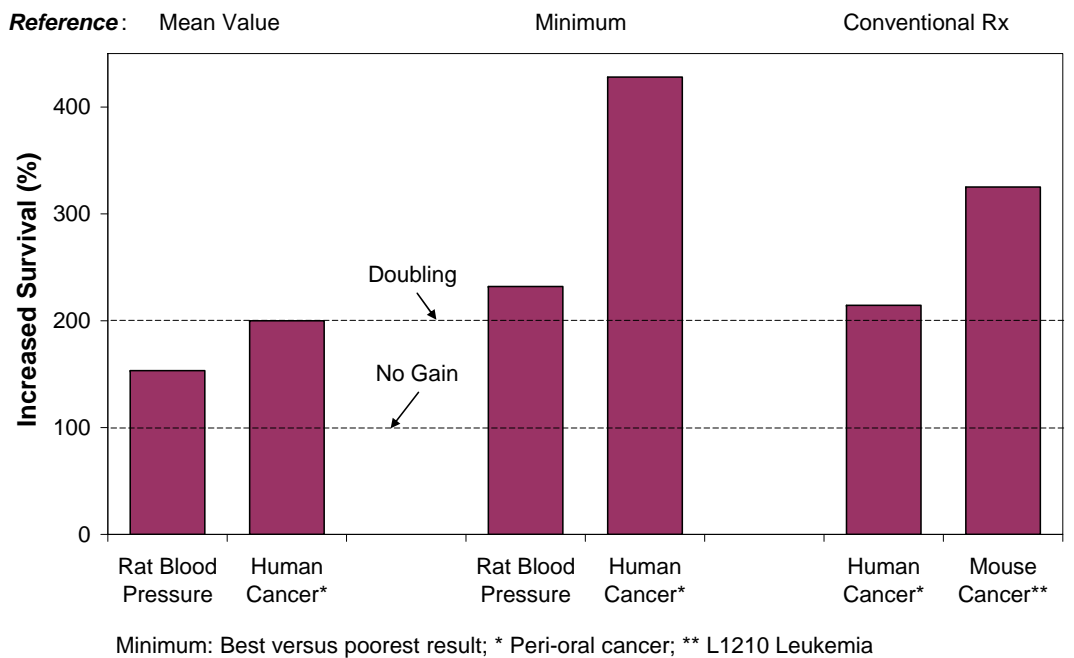

Fig. (6). Substantial gains from chronotherapy. (C) Halberg [13, 14].

coQ10 Treatment (Rx) Lowers the MESOR (M) of Blood Pressure (BP) of a Clinically Healthy Normotensive Woman (GC, 55y)

N-of-1 Study with Weekly Changes in Timing of coQ10 Administration*
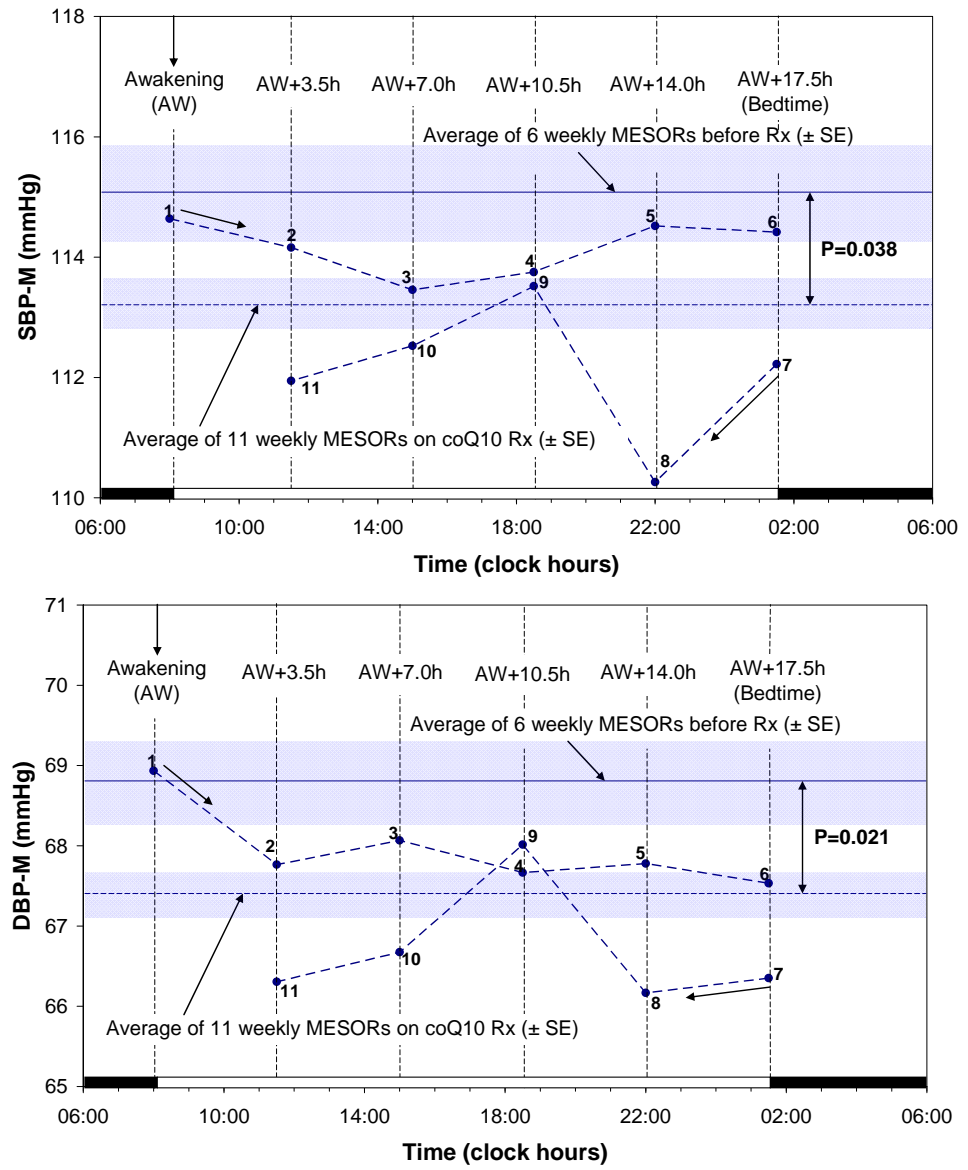

* At 6 different circadian stages, 3.5 hours apart during waking. Shaded areas represent mean \pm SE of 6 (before) and 11 (during coQ10 Rx) weekly MESORs, respectively.

P-value from Student t-test indicates lowering of MESOR of both systolic (S) and diastolic (D)

$\mathrm{BP}$, albeit NOT (yet?) in a circadian stage-dependent manner ( $\mathrm{P}>0.50$; not shown).

Fig. (7). Summary of effects on the MESOR (Midline Estimating Statistic Of Rhythm, M) of systolic (S) and diastolic (D) blood pressure (BP) assessed by self-surveillance by GC (F, 55y), who took coenzyme Q10 softgels (Q-Gel) supplementation (kindly provided by the Tishcon Corporation, Westbury, NY) at different times after awakening, namely upon awakening for one week, and at 3.5, 7, 10.5, 14 and 17.5 hours after awakening during consecutive weeks, while the 6 weeks of monitoring before the start of treatment served as reference. () Halberg. 

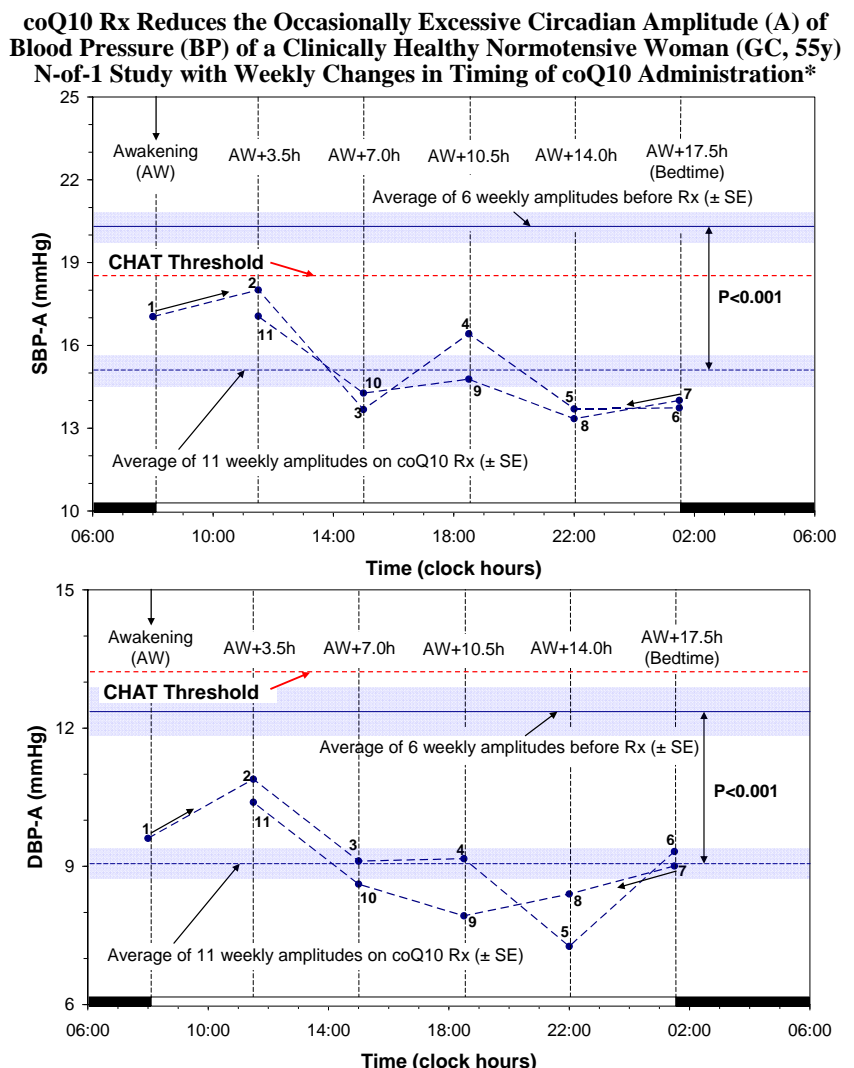

* At 6 different circadian stages, 3.5 hours apart during waking. Shaded areas represent mean \pm SE of 6 (before) and 11 (during coQ10 Rx) weekly circadian amplitudes, respectively.

CHAT: Circadian Hyper-Amplitude-Tension (overswing), a condition associated with an increase in cardiovascular disease risk larger than MESOR-Hypertension.

$\mathrm{P}$-value from Student $\mathrm{t}$-test indicates reduction in circadian amplitude of both systolic ( $\mathrm{S}$ ) and

diastolic (D) BP, in a circadian stage-dependent manner ( $\mathrm{P}<0.05$; not shown).

Fig. (8). Circadian time-dependence of the effect of coenzyme Q10 upon the circadian amplitude (A) of systolic (S) and diastolic (D) blood pressure (BP) of GC. Note that treatment eliminated a pre-existing CHAT (Circadian Hyper-Amplitude-Tension). (C) Halberg [16, 17].
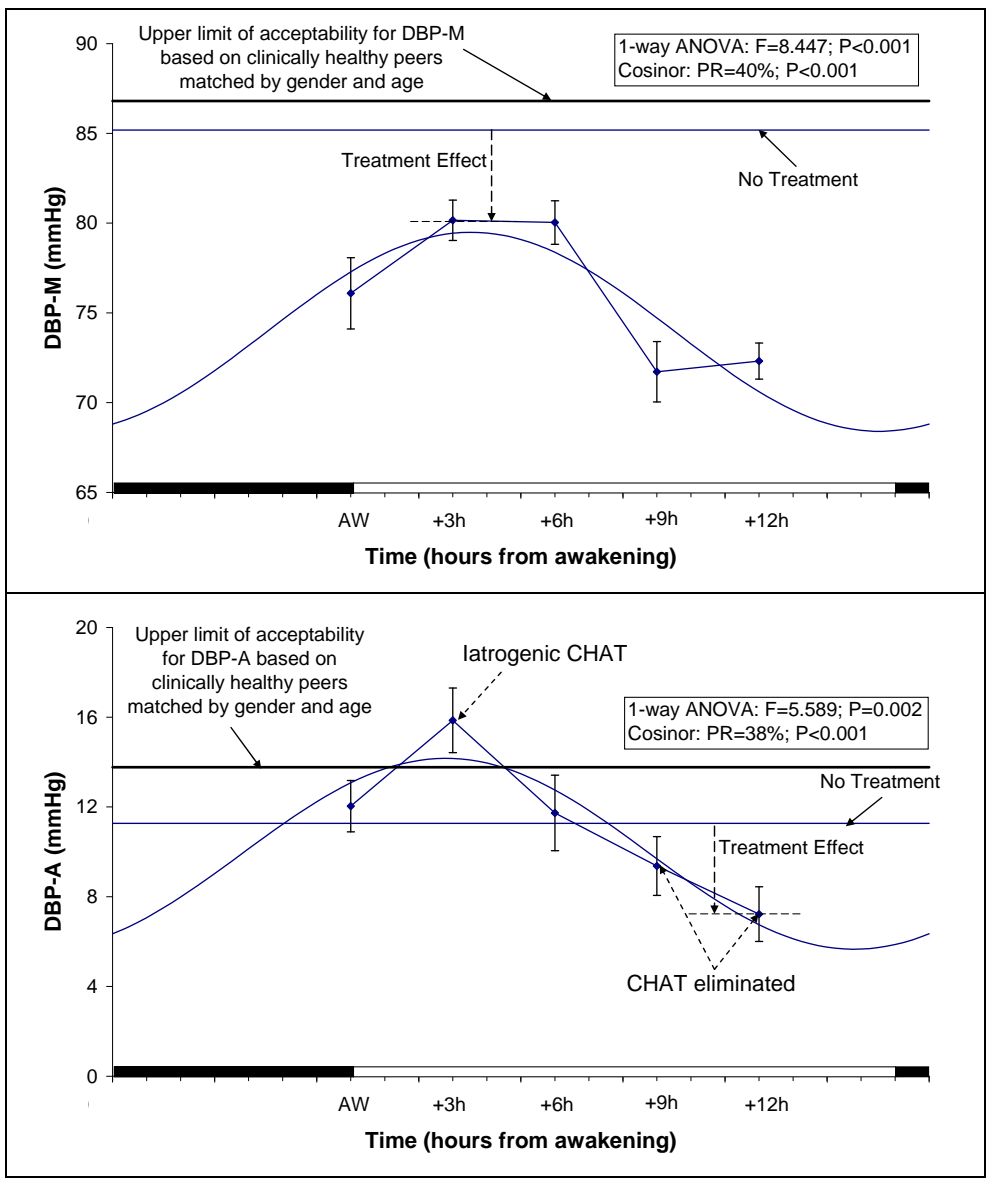


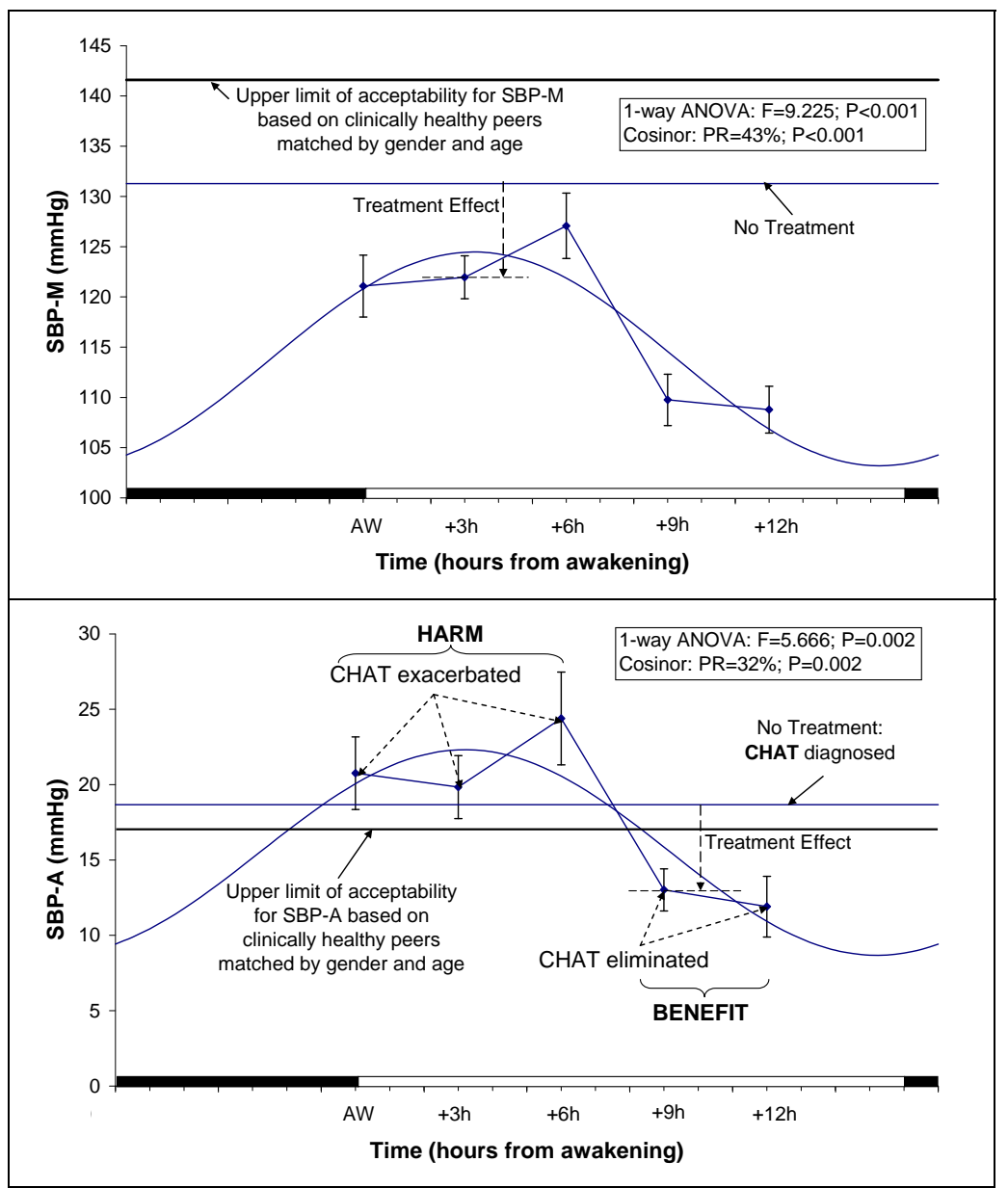

C Mild 7-day CHAT (left), exacerbated by Treatment (Rx) at the
wrong time (middle) but eliminated by $R x$ at the right time (right)

No Rx

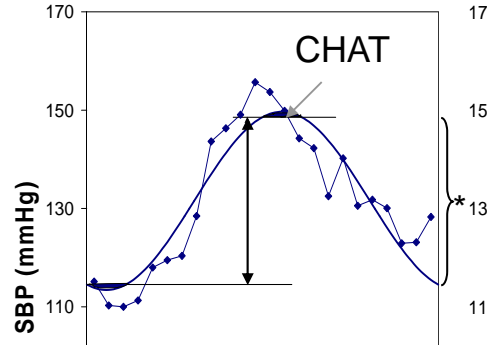

90 - Problem diagnosed:
CHAT
Rx 6h after AW

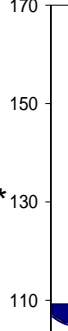

110

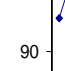

HARM:

Worse CHAT
Rx 12h after AW

BENEFIT:

CHAT Eliminated

150 - Risk of stroke reduced

Fig. 9. Contd.... 
Fig. 9. Contd....

D

Treatment Beneficial at Certain Other Times (9, 12 or 15 hours after awakening) can EXACERBATE a Pre-existing CHAT in Systolic Blood Pressure (SBP) and INDUCE CHAT in Diastolic Blood Pressure (DBP) when Given at the Wrong Time in Patient Su *
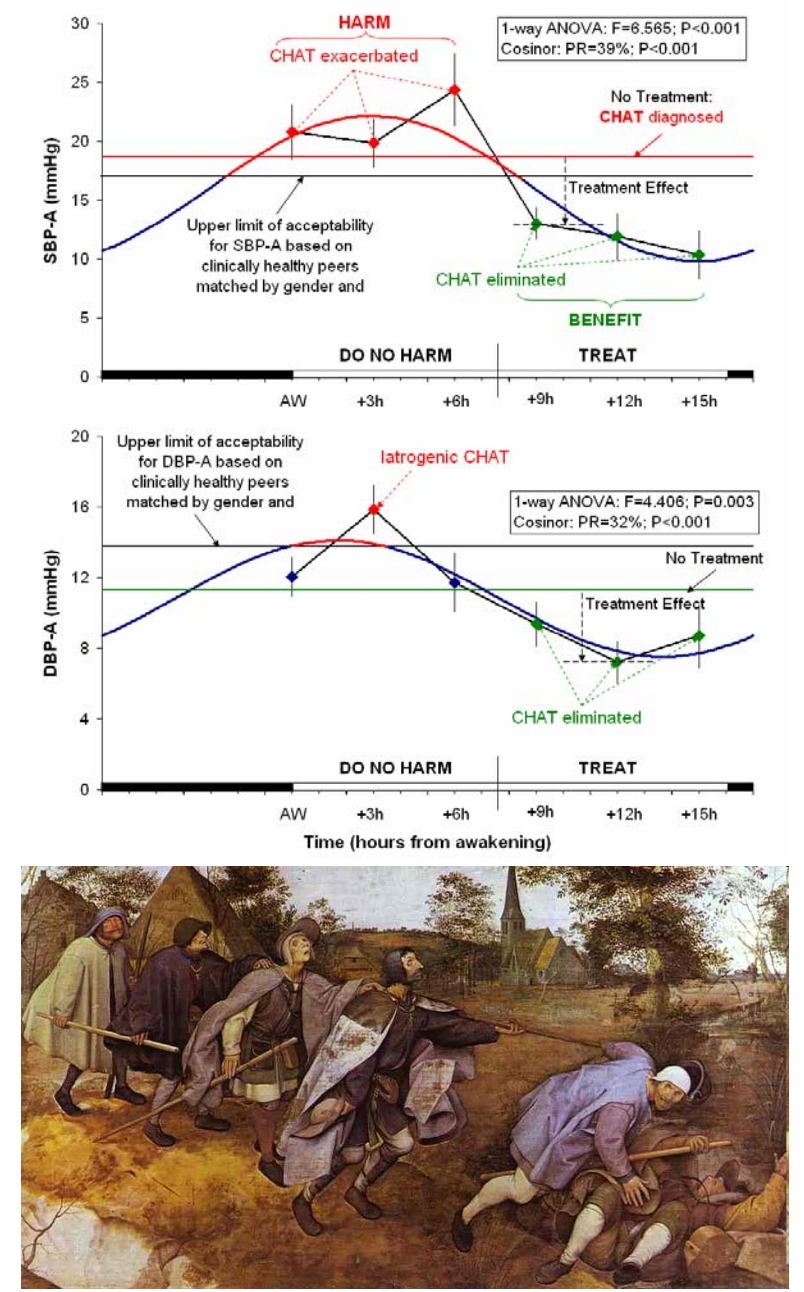

* Su, M, 66y, treated with Losartan $(50 \mathrm{mg})$ and hydrochlorothiazide $(12.5 \mathrm{mg})$. Each point represents 1 week of half-hourly around the-clock monitoring after $\sim 1$ month on a given treatment time. After this graph was completed, treatment at $A W+15 \mathrm{~h}$ yielded a SBP-A of $10.36 \pm 2.09 \mathrm{mmHg}$ and a DBP-A of $8.69 \pm 1.85 \mathrm{~mm} \mathrm{Hg}$. By cosinor, a circadian stage-dependent response to treatment could be shown to account, with statistical significance for both endpoints, for $39 \%$ and $32 \%$ of the overall variance, respectively.

Fig. (9A). Hyzaar induces CHAT in diastolic (D) blood pressure (BP) of one patient when given at some but not at other circadian stages. This figure is pertinent to large numbers of people worldwide, who are currently treated for high BP. They must realize that a popular drug, if prescribed without personalized surveillance, can harm by inducing a Vascular Variability Disorder (VVD), such as a circadian overswing or CHAT (Circadian Hyper-Amplitude-Tension). A change in the time when the drug is taken can make the same dose of the same drug in the same person beneficial rather than harmful or vice versa [21]. The drug can eliminate CHAT, a risk of stroke and other morbid events greater than a high BP, or induce it, depending on timing. Hyzaar was taken at one of 6 treatment times during waking, the schedule being changed monthly, with monitoring around the clock at 30-min intervals during the last week of each month. Differential effects on the MESOR (M) (top) and circadian amplitude (A) (bottom) of DBP. Differential effects of the same drug in the same dose in the same person occurring, all else being equal, as a function of the timing of the drug's use along the scale of 24 hours, yet without an account for any infradian dynamics that should prompt repeated administrations at the time found optimal only or in the course of a second cycle of variations in treatment times. Continued surveillance is best best implemented to avoid harm. Study by Dr. Yoshihiko Watanabe. () Halberg. (9B). Hyzaar exacerbates a preexisting CHAT in the systolic (S) blood pressure (BP) of Figure 9a's patient when given in the morning and eliminates this pre-existing VVD when given in the evening [21]. Study by Dr. Yoshihiko Watanabe. (C) Halberg. (9C). Without surveillance, we leave it up to chance and ignore whether we do harm at one time, shown in black (middle), while we can be beneficial at another time (right). (C) Halberg. (9D). If timing is not tested, insofar as vascular variability disorders (VVDs) are concerned (see Figs. 9A-C), the blind (care providers) lead the blind (care recipients) (bottom), showing the status quo. The painting at the bottom by Pieter Brueghel, "The Parable of the Blind Leading the Blind", is reproduced by kind permission of the Fototeca della Soprintendenza of the BAS PSAE and of the Polo Museale of the City of Naples, in order to emphasize that CHAT is silent to both the caregiver acting on the basis of a conventionally interpreted (chronobiologically uninterpreted) 24-hour blood pressure as well as to the majority of providers treating on the basis of single measurements in their office. (C) Halberg. 


\section{Continuous Surveillance of Hypotensive Therapy for both \\ Desired (MESOR, top) and Undesired (Circadian Amplitude, bottom) Effects by Sequential Timing of Blood Pressure Dynamics}

Changing of Timing of Medication ( $\Delta \mathrm{Rx})$ during Consecutive Spans
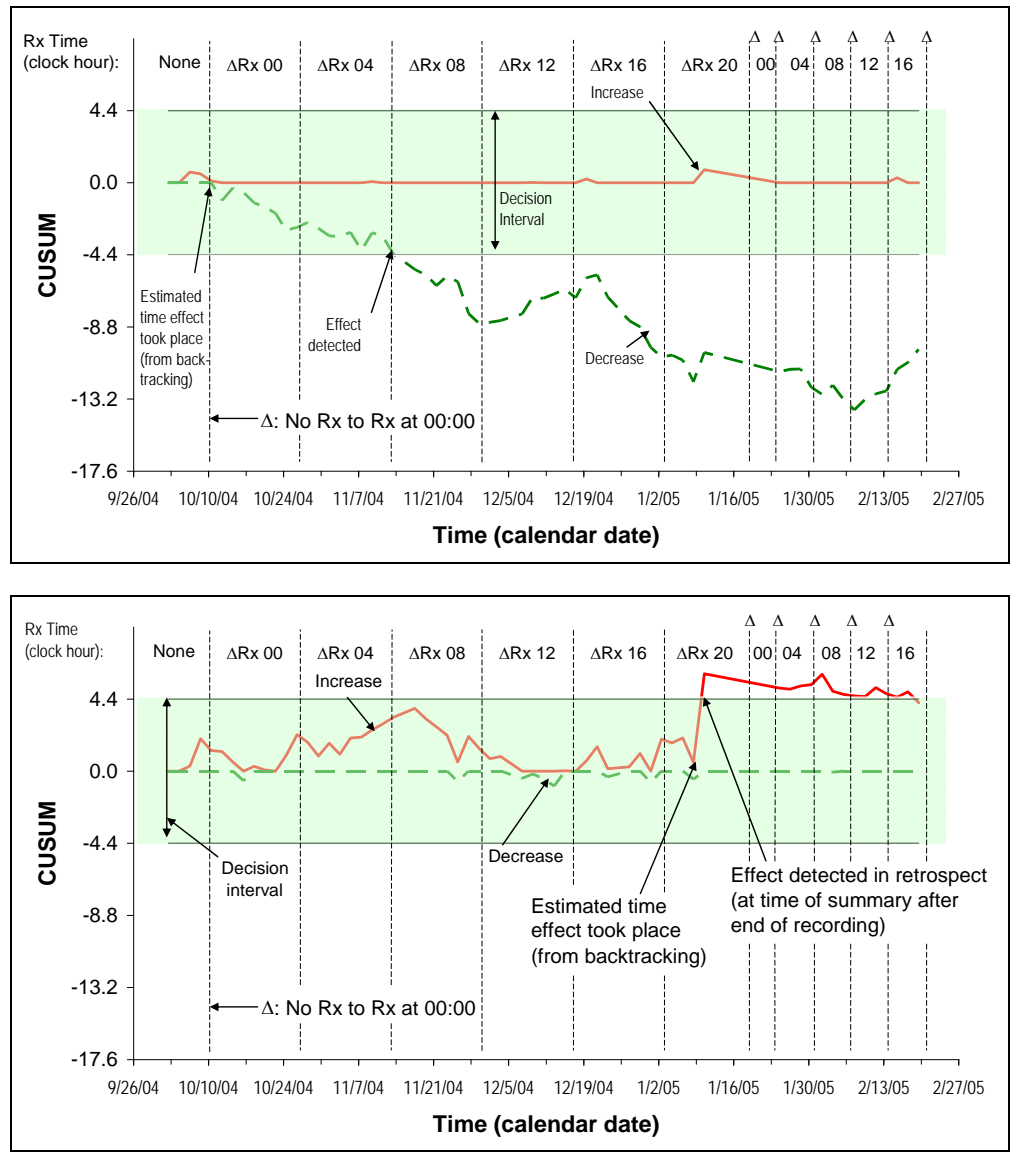

Fig. (10). The Fig. (9) results followed-up on a study wherein immediately after the diagnosis of MESOR-hypertension, the time when a hypotensive drug was taken was systematically varied as an empirical approach to chronotherapy, for a first cycle of treatment (Rx) times at about 17-day intervals, with continued half-hourly monitoring of blood pressure (BP) and with more frequent Rx changes in a second confirmatory cycle of Rx time. This procedure is recommended for use at the time of diagnosis of each new case whenever the first Rx time, chosen to precede the early rise in any excessive BP MESOR (M, top) and/or amplitude (A, bottom), does not provide the desired response of all circadian parameters involved. Top: Successful Rx of MESOR-hypertension can be assessed by a self-starting cumulative sum (CUSUM) control chart. To optimize his hypotensive Rx, a just-diagnosed 24-year-old individual (TT) switched his Rx first every 17 days by 4 hours and then mostly at shorter intervals. Note statistically significant decrease in BP-M, evidenced by the breakout outside the decision interval of the negative CUSUM line. With continued Rx, the BP-MESOR leaves the decision interval, indicating a statistically significant decrease in overall BP. Note, however, that with $\mathrm{Rx}$ at noon, the slope changes direction. Bottom: Changing timing of Rx during consecutive spans shows risk of iatrogenic CHAT. It is important to ascertain that no vascular variability disorder (VVD, e.g., CHAT) is being induced by inappropriate timing of Rx. In TT, Rx in the evening was associated with an iatrogenic increase in the circadian BP-A. We must always check whether the risk of MESOR-hypertension may not have been traded for the even higher risk of stroke that CHAT represents. Iatrogenic CHAT, induced by Rx at 20:00 daily, is silent to measurement restricted to office visits. Some individuals may have traded benefit (lowering of the BP-MESOR) for something worse (CHAT). This danger applies to some hypertensives (who tend to have a large circadian BP-A) to whom Rx time is not specified by the care provider, as was the case for TT (or is specified for the morning for somebody who could benefit from Rx in the evening), as seen in Fig. (9). Rx time, if it is not fully acceptable, with a first specified timing, is best individualized, so that other-wise undetected harm is revealed and the condition is removed by optimized timing achieved with as-one-goes sequential analysis. (c) Halberg.

\section{DISCUSSION}

With currently available instrumentation, it is seen that the same drug in the same dose given to the same person at different circadian stages can have opposite effects, i.e., harm or benefit [21]. At one circadian stage, it can be beneficial by lowering a very high risk of stroke and of other lifethreatening illnesses associated with a circadian overswing of $\mathrm{BP}$, whereas when it is given a few hours earlier or later, it induces or exacerbates this risk (Fig. 9).

Like the timing of drugs and nutrition, that of nutriceuticals requires objective inferential statistical study on the given individual. Reviews of the timing of drugs are available [22-24], yet their complete list is beyond the scope of this note. So is the important mapping of mechanisms in- 


\begin{tabular}{|c|}
\hline 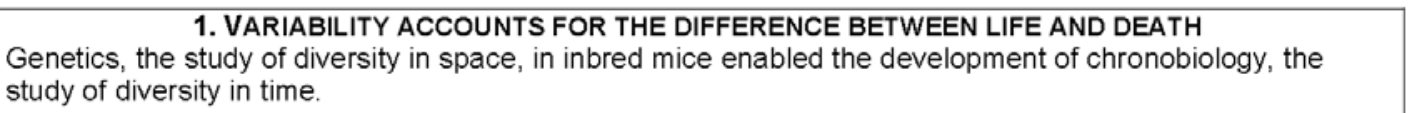 \\
\hline $\begin{array}{l}\text { Both are important since: } \\
\text { In response to the same stimulus such as noise, } \\
\text { - one audiogenically susceptible inbred strain of mice may respond with convulsions and death; but } \\
\text { - another audiogenically resistant strain may not respond to the same noise and will survive } \\
\text { as studied by genetics }\end{array}$ \\
\hline $\begin{array}{l}\text { In response to the same stimulus such as noise, } \\
\text { - one group of audiogenically susceptible mice may respond with convulsions and death when exposed at a } \\
\text { vulnerable time; but } \\
\text { - another group of the same audiogenically susceptible mice can be prevented from convulsions and death } \\
\text { and will survive, when exposure is targeted at a resistant time; i.e., by shielding in time } \\
\text { as studied by chronobiology }\end{array}$ \\
\hline $\begin{array}{r}\text { Next, nonphotic cycles were found to charac } \\
\text { - sudden cardiac death, suicide, crime, war } \\
\text { as s }\end{array}$ \\
\hline $\begin{array}{l}\text { 2. ORIGINS: } \\
\text { - Study of the quantitative rules of plant hybridization began in a pea patch in Brno, now in the Czech } \\
\text { Republic, and developed into genetics } \\
\text { - Study of the quantitative and inferential rules of variability in blood eosinophil counts in inbred mice began } \\
\text { in Minneapolis, USA, and developed into chronobiology } \\
\text { - These sciences study diversity: } \\
\text { - genetics in space } \\
\text { - chronobiology in time } \\
\text { The diversity of the cosmos embedded in us led to chronomics, the study of aeolian yet congruent } \\
\text { transdisciplinary time series around us, e.g., by chronobiologically interpreted } 7 \text {-day/24-hour and much } \\
\text { longer multidecadal, physiological and archival monitoring implemented in Brno, Minneapolis and the } \\
\text { broader project on The BIOsphere and the COSmos, BIOCOS }\end{array}$ \\
\hline
\end{tabular}

\section{Scheme 1.}

volved in abnormal nutrition [25]. We here emphasize the importance of continuous surveillance, when multi-purpose marker rhythms are available. Blood pressure and heart rate monitoring, for instance, serves as a marker of timing for the treatment of vascular variability disorders (VVDs), in relation to any excess in MESOR and/or circadian amplitude. It yields endpoints for validation of both a desired and/or an undesired effect(s) (Fig. 7-8) upon the MESOR and/or circadian amplitude. The recognition of otherwise often silent side effects such as undue disease risk, Fig. (10), is a great merit. In an era in which we monitor garages continuously and laboratory animals by telemetry to develop drugs, the broad field of nutriceuticals also awaits optimization by timing with continued surveillance. It cannot be overemphasized that abnormality in the human circulation cannot be reliably diagnosed without its presence in several 7-day monitoring sessions. Otherwise, conditions that can also occur for up to a few days in everyday life can be misdiagnosed as VVDs. It may not be avoided, and it is not necessarily harmful, as long as it is not consistent. BP elevations to hypertensive values can occur in the core of very friendly discussions, as can other changes in BP dynamics. Hence, it must be emphasized that VVDs cannot be diagnosed, not even in a single 7-day monitoring span, and can be called anomalies or abnormalities (VVAs) until their week-to-week consistency suggests that they are VVDs or vascular variability syndromes (VVSs) when more than a single VVD are present.

Scholars interested in nutriceuticals, like those in all other fields of human endeavor, including theoretical and applied physics, cannot dispense with the study of variabil- ity, which holds the key to all changes as a function of time as the indispensable control information. Without measuring variability in us, among us and around us, we are likely to commit blunders that can be avoided once variability has been mapped. It is not trivial, as seen in Scheme 1. It is true that penicillin was found without studying variability, just as much physics was learned before the atom was split. The latter feat released much energy, incomparably more than was possible earlier. Likewise, splitting the normal range into rhythms, like fission, and learning how internal and external rhythms interact, like fusion, can be anticipated to yield much useful information, also on nutriceuticals.

\section{CONCLUSIONS}

The same calorie is not utilized the same way at breakfast as it is at dinner.

The same dose of a drug that kills most animals at one time may be survived by most animals 12 hours earlier or later.

The same hypotensive drug can induce or exacerbate a circadian overswing, a high risk factor for stroke, or eliminate a pre-existing overswing, i.e., in the same person in the same dose it can be harmful at one body time or beneficial at another time.

We must not fly blind in administering nutriceuticals.

\section{REFERENCES}

[1] Arble DM, Bass J, Laposky AD, Vitaterna MH, Turek FW. Circadian timing of food intake contributes to weight gain. Obesity 2009; 17: 2100-2. 
[2] Arble DM, Goldschmidt C, Vitaterna MH, Turek FW. Circadian eating and sleeping patterns in the night eating syndrome. Sleep 2009; 32: A61.

[3] Halberg F. Protection by timing treatment according to bodily rhythms: an analogy to protection by scrubbing before surgery. Chronobiologia 1974; 1(Suppl. 1): 27-68.

[4] Halberg F, Haus E, Cornélissen G. From biologic rhythms to chronomes relevant for nutrition. In: Marriott BM, Ed. Not eating enough: Overcoming underconsumption of military operational rations. Washington DC: National Academy Press, 1995; pp. 361372.

http://books.nap.edu/books/0309053412/html/361.html\#pagetop

[5] Goetz FC, Bishop J, Halberg F, et al. Timing of single daily meal influences relations among human circadian rhythms in urinary cyclic AMP and hemic glucagon, insulin and iron. Experientia (Basel) 1976; 32: 1081-4.

[6] Graeber RC, Gatty R, Halberg F, Levine H. Human eating behavior: preferences, consumption patterns and biorhythms. Report No.: NATICK/TR-78/022. Technical Reports: U.S. Army, 1978, 287.

[7] Nelson W, Cadotte L, Halberg F. Circadian timing of single daily "meal" affects survival of mice. Proc Soc Exp Biol (NY) 1973; 144: 766-9.

[8] Nozawa M, Sugimoto K, Ohmori M, Ando H, Fujimura A. Dosing time-dependent effect of temocapril on the mortality of strokeprone spontaneously hypertensive rats. J Pharmacol Exp Ther 2006; 316: 176-81.

[9] Kumagai Y, Cornélissen G, Fujimura A, et al. Chronotherapy of vascular variability disorders: a challenge for the clinic. Proceedings of 1st International Workshop, Physiology of adaptation and quality of life: problems of traditional medicine and innovation, People's Friendship University of Russia; Moscow, Russia. May 14-16 2008; pp. 404-7.

[10] Halberg F, Cornélissen G, Wang ZR, et al. Chronomics: circadian and circaseptan timing of radiotherapy, drugs, calories, perhaps nutriceuticals and beyond. J Exp Ther Oncol 2003; 3: 223-60.

[11] Halberg Francine, Cornélissen G, Halberg F, et al. BIOCOS project. Reasons for a protocol for radiation treatment aimed at exploiting weekly rhythms. In: Halberg F, Kenner T, Fiser B, Siegelova J, Eds. Proceedings, Noninvasive Methods in Cardiology; Brno, Czech Republic, 2008 Oct 4-7; pp. 63-73. http://web.fnusa. cz/files/kfdr2008/sbornik_2008.pdf

[12] Cornélissen G. Profiles in Science: Franz Halberg. J Exp Ther Oncol 2008; 7 (4): 257-62.

[13] Halberg F, Cornélissen G, Schwartzkopff O. Introduction: Time, diagnostics, and therapeutics -- beyond circadian marker rhythmguided treatment. In: Youan BC, Eds. Chronopharmaceutics: Science and technology for biological rhythm-guided therapy and prevention of diseases; Hoboken, NJ: Wiley 2009; pp. xvii-xxxii.
[14] Halberg F, Cornélissen G, Schwartzkopff O. Implications and applications of circadian susceptibility rhythms: chronomics and anesthesia. In: Youan BC, Eds. Chronopharmaceutics: Science and technology for biological rhythm-guided therapy and prevention of diseases; Hoboken, NJ: Wiley 2009; pp. 217-55.

[15] Cornélissen G, Halberg F. Treatment with open eyes: markersguided chronotheranostics. In: Youan BC, Eds. Chronopharmaceutics: Science and technology for biological rhythm-guided therapy and prevention of diseases; Hoboken, NJ: Wiley 2009; pp. 257323.

[16] Cornélissen G, Halberg F, Schwartzkopff O, et al. Coenzyme-Q10 effect on blood pressure variability assessed with a chronobiological study design. Noninvasive Methods in Cardiology; Brno Czech Republic Abstract book, 2005; s.10.

[17] Halberg F, Cornélissen G, Singh RB, et al. Chronobiology, chronomics and N-of-1 tests of timing coenzyme Q10. In: Gvozdjakova A, Eds. Mitochondrial medicine: mitochondrial metabolism, diseases, diagnosis and therapy; Dordrecht: Kluwer; 2008; pp. 5592.

[18] Jassim AD, Brown J, Hrushesky W, Cornélissen G, Halberg F Reduction by ubiquinone (CoQ10) of doxorubicin (D) toxicity in LOU rats bearing an immunocytoma. Chronobiologia 1983; 10: 135.

[19] Singh RB, Kartik C, Otsuka K, Pella D, Pella J. Brain-heart connection and the risk of aspects of health and disease. Biomed Pharmacother 2002; 56 (Suppl 2): 257-65.

[20] Singh RB, Pella D, Otsuka K, Halberg F, Cornélissen G. New insights into circadian aspects of health and disease. J Assoc Physicians India 2002; 50: 1416-25.

[21] Watanabe Y, Cornélissen G, Halberg F, et al. Harm vs. benefit from losartan with hydrochlorothiazide at different circadian times in MESOR-hypertension or CHAT. In: Halberg F, Kenner T, Fiser B, Siegelova J, Eds. Proceedings, Noninvasive Methods in Cardiology; Brno, Czech Republic, pp. 2008 Oct 4-7; 149-67. http://web.fnusa.cz/files/kfdr2008/sbornik_2008.pdf

[22] Lemmer B. The importance of circadian rhythms on drug response in hypertension and coronary heart disease -- from mice and man. Pharmacol Ther 2006; 111: 629-51.

[23] Ohdo S. Changes with toxicity and effectiveness with timing of drug administration: implications for drug safety. Drug Saf 2003; 26 (14): 999-1010.

[24] Moss RW. Chronobiology and chronotherapy. Weekly CancerDecisions.com Newsletter \#85 05/30/03. http://www.alkalizeforhealth. net/1.chronobiology.htm

[25] Goel N, Stunkard AJ, Rogers NL, et al. Circadian rhythm profiles in women with night eating syndrome. J Biol Rhythms 2009; 24: 85-94. 